\title{
Impact of Water on the Cis-Trans Photoisomerization of Hydroxychalcones
}

\author{
Yoann Leydet, ${ }^{* \dagger}$ Pinar Batat, ${ }^{\ddagger}, \S$ Gediminas Jonusauskas, ${ }^{\S}$ Sergey Denisov, ${ }^{\ddagger, \S}$ João Carlos Lima, ${ }^{* \dagger}$
} A. Jorge Parola, ${ }^{\dagger}$ Nathan D. McClenaghan, ${ }^{\ddagger}$ and Fernando Pina ${ }^{\dagger}$

${ }^{\dagger}$ REQUIMTE, Departamento de Química, Faculdade de Ciências e Tecnologia, Universidade Nova de Lisboa, 2829516 Caparica, Portugal

${ }^{\ddagger}$ Institut des Sciences Moléculaires (ISM), CNRS UMR 5255, and ${ }^{\S}$ Laboratoire Ondes et Matière d'Aquitaine (LOMA), CNRS UMR 5798, Université de Bordeaux, F 33400 Talence, France

\begin{abstract}
The photochromism of a 2 hydroxychalcone has been studied in $\mathrm{CH}_{3} \mathrm{CN}$ and $\mathrm{H}_{2} \mathrm{O} / \mathrm{CH}_{3} \mathrm{OH}(1 / 1, \mathrm{v} / \mathrm{v})$, as well as in analogous deuterated solvents using steady state (UV-vis absorption, ${ }^{1} \mathrm{H}$ and ${ }^{13} \mathrm{C}$ NMR) and time resolved (ultrafast transient absorption and nanosecond flow flash photolysis) spectroscopies. Whereas the irradiation of trans chalcone $(\mathbf{C t})$ under neutral $\mathrm{pH}$ conditions leads to the formation of the same final chromene derivative (B) in both media, two distinct photochemical mechanisms are proposed in agreement with thermodynamic and kinetic properties of the chemical reaction network at the ground state. Following light excitation, the first steps are identical in acetonitrile and aqueous solution: the Franck-Condon excited state rapidly populates the trans chalcone

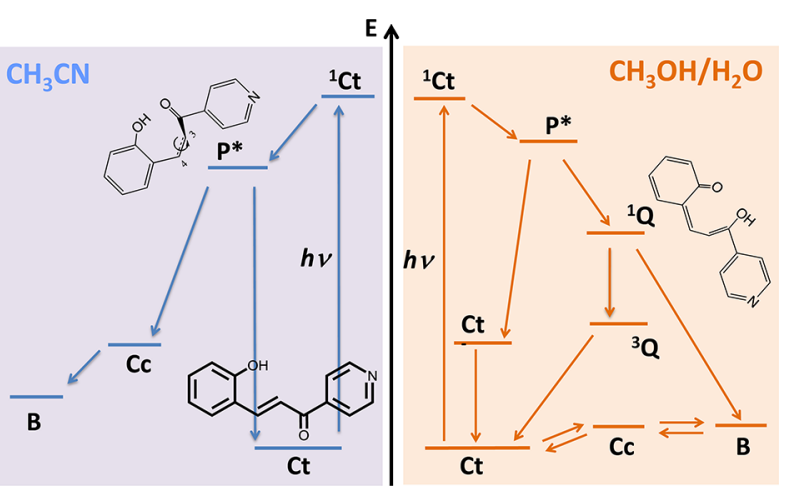
singlet excited state ${ }^{1} \mathrm{Ct}^{*}$ (LE), which evolves into a twisted state ${ }^{1} \mathbf{P}^{*}$. This excited state is directly responsible for the photochemistry in acetonitrile in the nanosecond time scale $(16 \mathrm{~ns})$ leading to the formation of cis chalcone (Cc) through a simple isomerization process. The resulting cis chalcone evolves into the chromene $\mathbf{B}$ through a tautomerization process in the ground state $(\tau=10 \mathrm{~ms})$. Unlike in acetonitrile, in $\mathrm{H}_{2} \mathrm{O} / \mathrm{CH}_{3} \mathrm{OH}(1 / 1, \mathrm{v} / \mathrm{v})$, the $\mathbf{P} *$ state becomes unstable and evolves into a new state attributed to the tautomer ${ }^{1} \mathbf{Q}^{*}$. This state directly evolves into $\mathbf{B}$ in one photochemical step through a consecutive ultrafast tautomerization process followed by electrocyclization. This last case represents a new hypothesis in the photochromism of 2 hydroxychalcone derivatives.
\end{abstract}

\section{INTRODUCTION}

Chalcones constitute an important class of compounds with applications in medical sciences, ${ }^{1}$ biotechnology, ${ }^{2}$ and photo chemistry, because of their photochromism based on a transcis isomerization process. ${ }^{3}$ Within this family of compounds, the natural 2 hydroxyderivatives are particularly interesting because they can promote the formation of the flavylium cation and the quinoidal base of anthocyanins, a family of pigments responsible for most of the red and blue colors appearing in flowers and fruits. ${ }^{4}$ Anthocyanins are involved in a network of chemical reactions including proton transfer, hydration, tautomerization, and cis-trans isomerization. ${ }^{5}$ The complexity of this natural network has been reproduced by flavylium salts, synthetic analogues of anthocyanins, leading to efficient and versatile photochromic systems depending on $\mathrm{pH}$ and light inputs. ${ }^{6}$

The network of chemical reactions occurring in flavylium compounds is shown in Scheme 1 for 2 phenyl 1 benzopyry lium (structurally, the simplest flavylium). In aqueous solution, at sufficiently acidic $\mathrm{pH}$ values $(\mathrm{pH}<1)$, the flavylium cation, $\mathrm{AH}^{+}$, is the more stable and dominant species. As the $\mathrm{pH}$ is increased $(\mathrm{pH}>2)$, the flavylium cation undergoes hydration to give the hemiketal $\mathbf{B}$ form, which, in turn, can tautomerize to form a cis chalcone $(\mathbf{C c})$. This species can further undergo isomerization to the trans chalcone $(\mathbf{C t})$ form. The system can proceed forward and backward by the action of $\mathrm{pH}$ and light and has been used as a "write-lock-read-unlock-erase" molecular switching system ${ }^{7}$ and to mimic some elementary properties of neurons. ${ }^{8}$ The photoisomerization observed for 2 hydroxychalcone derivatives has been widely studied, revealing a relationship between the quantum yield of isomerization and the chemical structure, viscosity, and polarity of the medium. ${ }^{6}$ Despite the apparent simplicity of photochemical processes, the ubiquity of systems displaying such reactions (e.g., olefins, stilbenes, azobenzenes, amides, enols) has led to a large variety of mechanisms accounting for the elementary processes after photoexcitation. ${ }^{9}$ Some parallels can be drawn between chalcones ${ }^{10}$ and other trans-cis photoisomerization model compounds (such as stilbene) ${ }^{11}$ and perhaps even more so with 
Scheme 1. Network of Chemical Reactions in Acidic/Neutral Medium Connecting trans 2 Hydroxychalcone $(\mathrm{Ct})$ and 2 Phenyl 1 benzopyrylium $\left(\mathrm{AH}^{+}\right)$

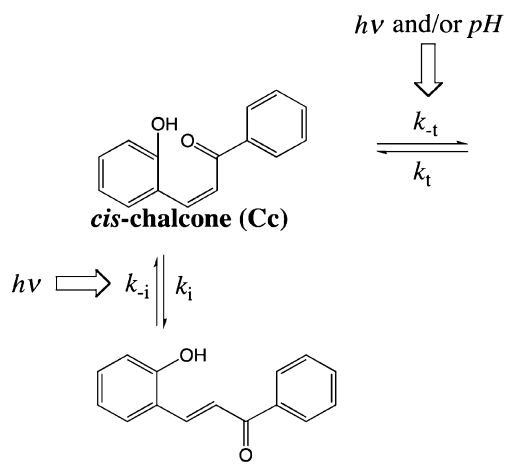

trans-chalcone $(\mathrm{Ct})$

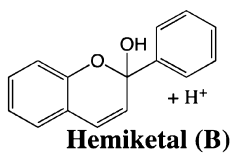

$k_{\mathrm{h}} \| k_{-\mathrm{h}} \Longleftarrow p H$

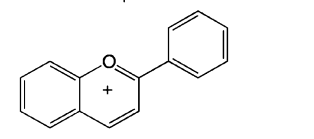

Flavylium cation $\left(\mathrm{AH}^{+}\right)$

compounds, such as dimethylaminobenzonitrile (DMABN), ${ }^{12}$ that contain donor/acceptor substituents across the double bond, which confers charge transfer character to the first excited state. However, unlike in the aforementioned photo chromic families, the photoisomerization reaction represents only one of the several processes taking place in the excited state, and competition between these processes can occur. Other photophysical processes have already been identified, such as the formation of various triplet states, electrocylization, or photoinduced proton transfer especially in aqueous medium. ${ }^{13}$ To the best of our knowledge, a comprehensive study including the effect of the solvent on the deactivation pathways of the first excited state and the identification of the first intermediate species after excitation leading to photo isomerization and other competing channels has never been published.

We recently demonstrated that the formation of a flavylium salt can be excluded or limited to extremely acidic conditions when an electron withdrawing substituent is inserted in the structure of the 2 hydroxychalcone skeleton. ${ }^{14,15}$ In this study, the absence of a flavylium cation facilitates not only the attribution of the processes involved in the photophysics of 2 hydroxychalcones but also the determination of the photo product of the reaction. We report a detailed study on the photophysics and photochemistry of a 2 hydroxypyridinechal cone (compound 1, Scheme 2). The aim of this work was to

\section{Scheme 2. Structure and Numbering of Compound $1^{a}$}

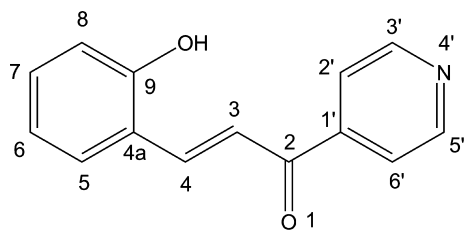

${ }^{a}$ Notation of the flavylium cation instead of the chalcone ions was used to simplify the discussion.

characterize the photochromism of 2 hydroxychalcones, from the formation of the excited state responsible for the isomerization process to the identification of the photoproduct. The solvent dependence of the photochemistry was studied first in an organic solvent $\left(\mathrm{CH}_{3} \mathrm{CN}\right)$ and then in an aqueous solution under neutral conditions $\left(\mathrm{H}_{2} \mathrm{O} / \mathrm{CH}_{3} \mathrm{OH}, 1 / 1\right.$, v/v), as

well as in the analogous deuterated mixture $\left(\mathrm{D}_{2} \mathrm{O} / \mathrm{CD}_{3} \mathrm{OD}, 1 /\right.$ $1, \mathrm{v} / \mathrm{v})$.

\section{EXPERIMENTAL SECTION}

Instrumentation and Measurements. Compound 1 was available from previous studies. ${ }^{14}$ Solutions were prepared using Millipore water and spectroscopic grade methanol and acetonitrile. Electronic absorption spectra were recorded on a Varian Cary 100 Bio or Shimadzu VC2501 PC spectropho tometer. Photochemical transformations were carried out using a medium pressure xenon/mercury arc lamp, and the excitation bands (254 and $365 \mathrm{~nm}$ ) were isolated with interference filters (Oriel). The incident light intensity was measured by ferrioxalate actinometry. ${ }^{16} \mathrm{NMR}$ spectra at $298.0 \mathrm{~K}$ were obtained on a Bruker AMX400 spectrometer operating at $400.13 \mathrm{MHz}\left({ }^{1} \mathrm{H}\right)$ and $100 \mathrm{MHz}\left({ }^{13} \mathrm{C}\right)$. Nanosecond laser flash photolysis experiments were run on an LKS.60 laser photolysis spectrometer from Applied Photophysics, using an Applied Photophysics SX20 stopped flow spectrometer to guarantee that all of the transient signals were reproducibly obtained with fresh solution and to avoid accumulation of photoproducts competing for light excitation.

Picosecond transient absorption spectra were recorded on a setup that was built as follows: A frequency tripled $\mathrm{Nd}$ :YAG amplified laser system ( 30 ps, $30 \mathrm{~mJ}$ at 1064 nm, $20 \mathrm{~Hz}$, Ekspla model PL 2143) output was used to pump an optical parametric generator (Ekspla model PG 401), producing tunable excitation pulses in the range of $410-2300 \mathrm{~nm}$. The residual fundamental laser radiation was focused in a high pressure Xe filled breakdown cell where a white light pulse for sample probing was produced. All light signals were analyzed by a spectrograph (Princeton Instruments Acton model SP2300) coupled with a high dynamic range streak camera (Hamamatsu C7700). Accumulated sequences (sample emission, probe without and with excitation) of pulses were recorded and treated with HPDTA (Hamamatsu) software to produce two dimensional maps (wavelength versus delay) of transient absorption intensity in the range of $300-800 \mathrm{~nm}$. Typical measurement error was better than $10^{-3}$ of the optical density (OD). Samples were equally studied on the subpicosecond time scale: This experiment was based on a femtosecond $1 \mathrm{kHz}$ Ti:sapphire system producing $30 \mathrm{fs}, 0.8 \mathrm{~mJ}$, laser pulses centered at $800 \mathrm{~nm}$ (Femtopower Compact Pro) coupled with an optical parametric generator (Light Conversion Topas C) and frequency mixers to excite samples at the maximum of the steady state absorption band. White light continuum (360$1000 \mathrm{~nm}$ ) pulses generated in a $2 \mathrm{~mm} \mathrm{D}_{2} \mathrm{O}$ cell were used as a probe. A variable delay time between excitation and probe pulses was obtained using a delay line with 0.66 fs resolution. The solutions were placed in a $1 \mathrm{~mm}$ circulating cell. White light signal and reference spectra were recorded using a two channel fiber spectrometer (Avantes Avaspec 2048 2). A self written acquisition and experiment control program in LabVIEW enabled the recording of transient spectra with an average error of less than $10^{-3}$ of the optical density for all wavelengths. The temporal resolution of the setup was better than 50 fs. A temporal chirp of the probe pulse was corrected by a computer program with respect to a Lawrencian fit of a Kerr signal generated in a $0.2 \mathrm{~mm}$ glass plate used in place of a sample. 


\section{RESULTS AND DISCUSSION}

Study in Acetonitrile. Irradiation of an equilibrated solution of compound $\mathbf{1}$ in acetonitrile at $365 \mathrm{~nm}$ was followed by UV-vis absorption spectroscopy (Figure 1A). The spectrum
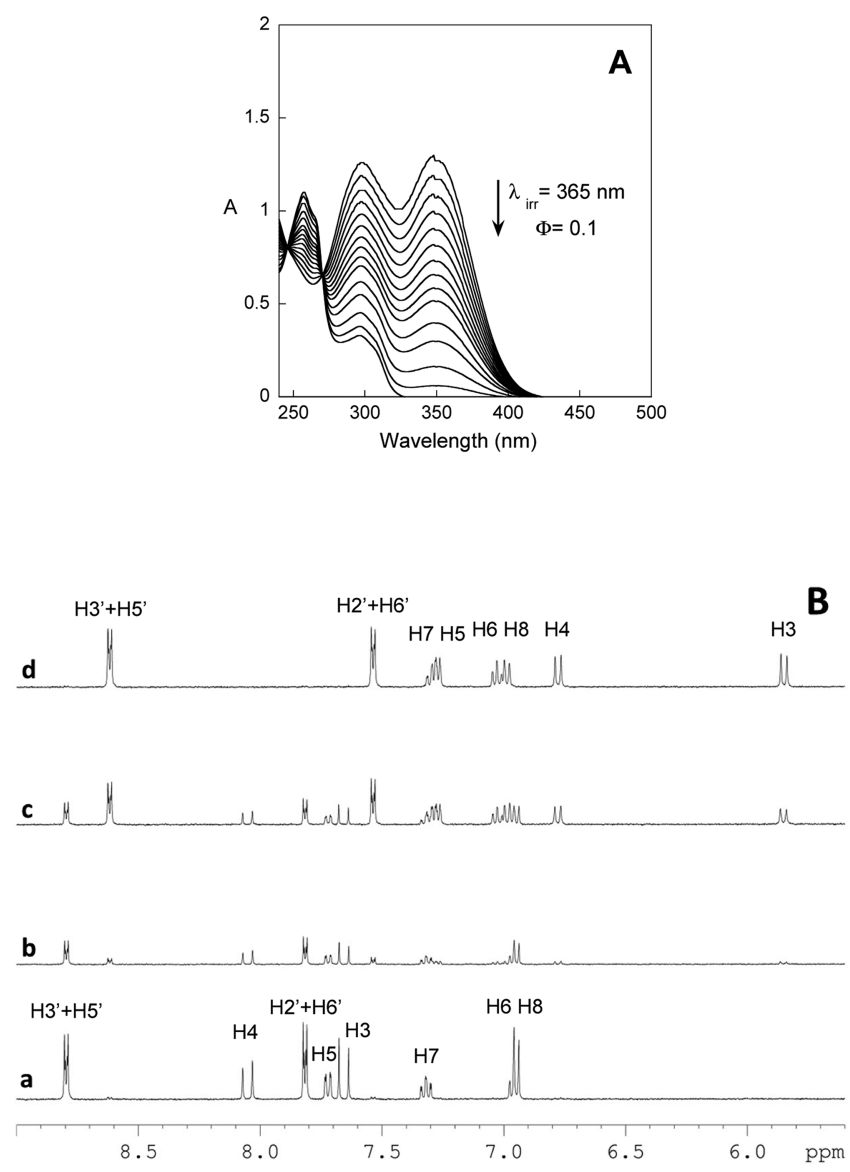

Figure 1. (A) Spectral variations upon continuous irradiation of compound $\mathbf{1}\left(C_{0}=1.1 \times 10^{-4} \mathrm{M}\right)$ in acetonitrile at $365 \mathrm{~nm}(\Phi=0.1)$. (B) ${ }^{1} \mathrm{H}$ NMR spectra $(400 \mathrm{MHz}, 300 \mathrm{~K})$ of 1 in $\mathrm{CD}_{3} \mathrm{CN}$ (a) at thermal equilibrium ( $>95 \% \mathbf{C t},<5 \% \mathrm{~B}),(\mathrm{b}, \mathrm{c})$ upon irradiation at 365 $\mathrm{nm}$, and (d) after reaching the photostationary state upon irradiation at $365 \mathrm{~nm}(100 \%$ B). Proton assignments for $\mathbf{C t}$ and $\mathbf{B}$ given in traces $\mathrm{a}$ and $\mathrm{d}$, respectively (according to the numbering of $\mathbf{C t}$ in Scheme 2 and assuming the same numbering for $\mathbf{B}$ ). of the initial solution (thermally equilibrated) is dominated by a broad band at $350 \mathrm{~nm}$ assigned to the absorption of the $\mathbf{C t}$ form. This band decreases upon irradiation until a photosta tionary state is reached, displaying two absorption bands with maxima at 254 and $300 \mathrm{~nm}$. The spectral variations are consistent with the disappearance of $\mathbf{C t}$ and concomitant formation of a photoproduct with a global quantum yield of 0.1 . The well defined isosbestic points (246 and $270 \mathrm{~nm}$ ) support the conclusion that $\mathbf{C t}$ is being converted into a single photoproduct.

${ }^{1} \mathrm{H}$ NMR spectra, obtained before, during, and after reaching the photostationary state (traces a-d in Figure 1B), show that the initially (thermally equilibrated) solution comprises two different species, one of which is predominant (>95\%). The predominant species displays a large coupling constant between protons $\mathrm{H} 3(\delta=7.65 \mathrm{ppm})$ and $\mathrm{H} 4(\delta=8.04 \mathrm{ppm})\left[{ }^{3} J(\mathrm{H} 3\right.$, $\mathrm{H} 4)=16.0 \mathrm{~Hz}]$, typical of the trans chalcone isomer, whereas the minor species $(<5 \%)$ is characterized by a smaller coupling constant $\left[{ }^{3} \mathrm{~J}(\mathrm{H} 3, \mathrm{H} 4)=9.8 \mathrm{~Hz}\right]$ (spectrum a). The initial $\mathbf{C t}$ is converted progressively into the minority species, which is also present in the initial solution (spectra $b-d$ ). Formation of additional species was not observed during irradiation or at the photostationary state. The nature of the photoproduct was unambiguously confirmed by NMR spectroscopy (HMBC experiments), where the $\mathrm{C} 2$ carbon presents a chemical shift in agreement with that of an $\mathrm{sp}^{3}$ carbon $(\delta=97.4 \mathrm{ppm}$, Figure S1, Supporting Information), which is only present in the hemiketal species, B. In contrast to aqueous solutions, for which complete recovery of $\mathbf{C t}$ is observed when solutions are allowed to equilibrate in the dark, species $\mathbf{B}$ is stable in acetonitrile, and only traces of $\mathbf{C t}$ can be found even after 3 days. Nevertheless, the photochemistry of $\mathbf{1}$ in $\mathrm{CH}_{3} \mathrm{CN}$ allows it to be described as a photochromic system because the recovery of $\mathbf{C t}$ can be achieved upon irradiation of $\mathbf{B}$ at $254 \mathrm{~nm}$ (data not shown).

Ultrafast transient absorption spectroscopy was employed to follow the evolution of the excited trans chalcone. The overall spectral evolution between 400 and $750 \mathrm{~nm}$ in $\mathrm{CH}_{3} \mathrm{CN}$ following $355 \mathrm{~nm}$ excitation is divided into two sequential steps (Figure 2).

Following excitation of $\mathbf{1}$, two distinct signals can be observed on a picosecond time scale (Figure 2, left). An initial positive absorption signal, assigned to a locally excited state
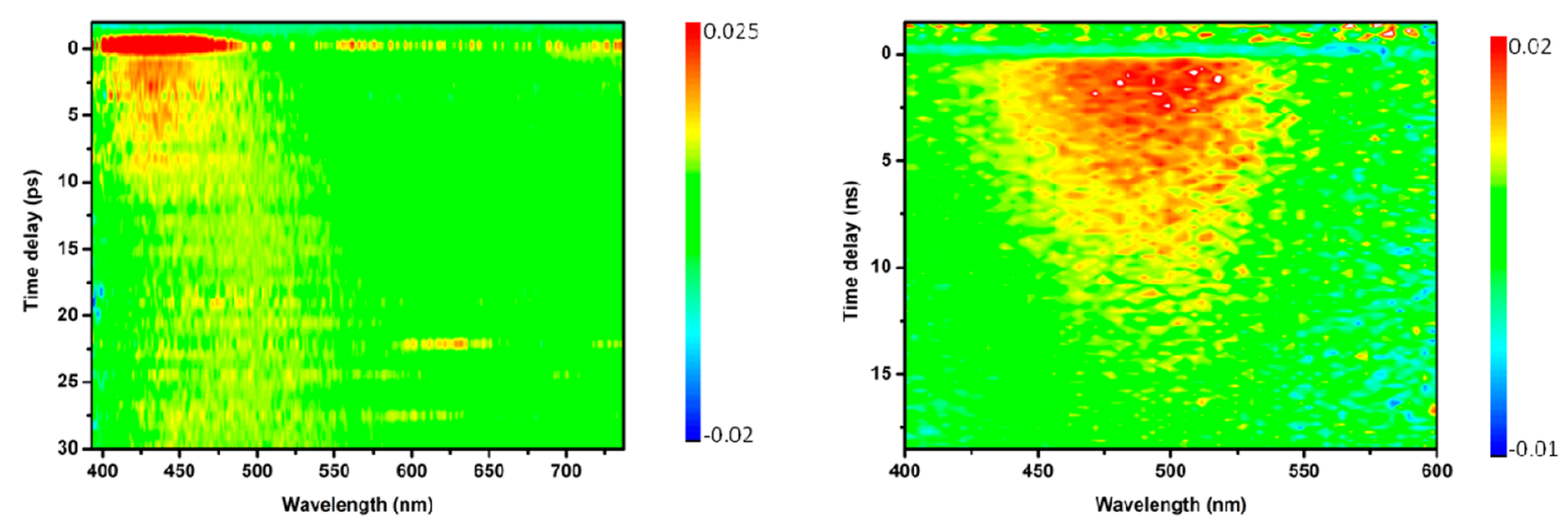

Figure 2. Ultrafast dynamics of 1 in $\mathrm{CH}_{3} \mathrm{CN}$. Transient absorption spectra with time delays of 0-30 ps (left) and 0-20 ns (right). Transient absorption bands were observed between 425 and $550 \mathrm{~nm}$ and decayed with a lifetime of $16 \mathrm{~ns}$. Colors correspond to $\Delta \mathrm{OD}$ values according to the included scales. 

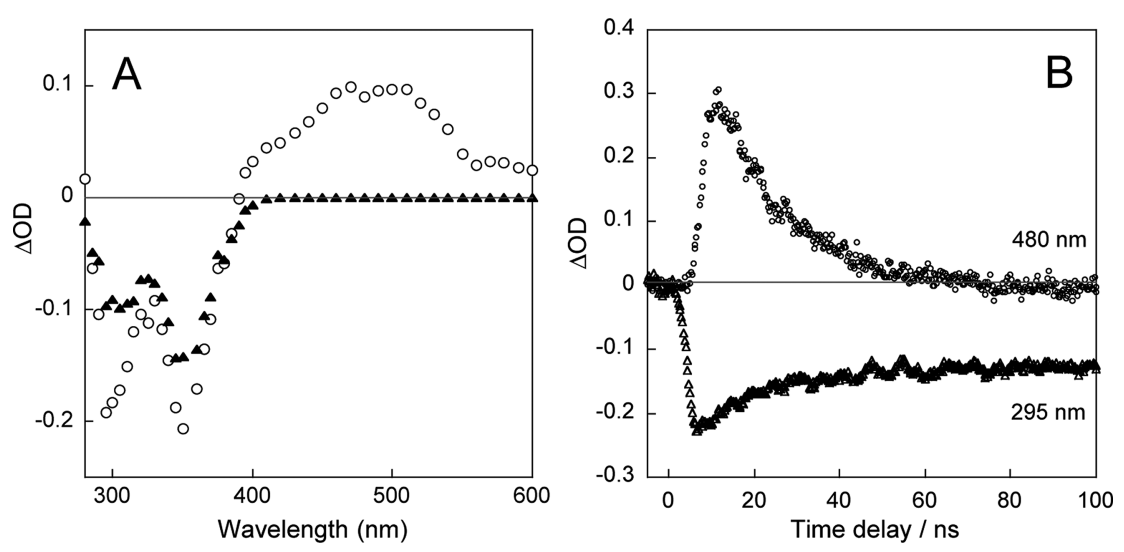

Figure 3. (A) Transient absorption spectra of $\mathbf{1}$ in $\mathrm{CH}_{3} \mathrm{CN}$ obtained by flash photolysis in air equilibrated solution $(\mathrm{O})$ immediately and ( $(\boldsymbol{\Delta}) 100 \mathrm{~ns}$ after the pulse. (B) Kinetic traces at $(O) 480$ and $(\Delta) 295 \mathrm{~nm}$.

(LE) absorbing at $420 \mathrm{~nm}$, appears immediately after the pulse and subsequently relaxes to a second state $(\mathbf{R})$, which presents a broad red shifted band extending between 400 and $550 \mathrm{~nm}$. The LE state decay time is identical to the formation time of the relaxed state $\mathbf{R}\left(\tau_{1}=2 \mathrm{ps}\right)$. The spectrum of $\mathbf{R}$ does not shift further, and the relaxed state decays on the nanosecond time scale $\left(\tau_{2}=16 \mathrm{~ns}\right)$ (see Figure 2 , right), at the same rate over the whole spectral region, indicating the presence of a single transient species. The absorption of $\mathbf{R}$ cannot be attributed to any molecule of the network in the ground state (Cc, B) because such species do not absorb at these wavelengths. Nanosecond flash photolysis $\left(\lambda_{\text {exc }}=355 \mathrm{~nm}\right)$ was employed to further follow the decay of the relaxed state $\mathbf{R}$ and characterize its relation with the ground state photo product formation. The time resolved absorption spectra immediately after the flash and after complete disappearance of $\mathbf{R}(100 \mathrm{~ns})$ are represented between 280 and $600 \mathrm{~nm}$ in Figure 3.

Whereas the spectrum recorded immediately after the nanosecond laser pulse is composed of the sum of $\mathbf{C t}$ bleaching (negative $\Delta \mathrm{OD}$ ) and transient absorption of $\mathbf{R}$ (positive $\Delta \mathrm{OD})$, the spectrum after $100 \mathrm{~ns}$ presents only a negative signal that is stable on the microsecond time scale. The recovery of $\mathbf{C t}$ is not complete, in agreement with the photoconversion of trans chalcone into photoproducts. Also, the final spectrum is different from that of pure $\mathbf{C t}$, confirming the presence of a photoproduct absorbing in the same region. The quantum yield for photoproduct formation can be crudely estimated to be 0.5 , based on the intensity of the persistent negative signal with respect to the initial intensity, immediately after excitation (see Figure $3 b$ ); however, the presence of a photoproduct absorbing in the same region can introduce a significant error in this value.

The pure $\mathbf{R}$ absorption can be obtained by subtracting the contribution of $\mathbf{C t}$ to the initial spectrum (Figure S2, Supporting Information), and the photoproduct can be obtained on subtracting the contribution of $\mathbf{C t}$ to the final spectrum (Figure S3, Supporting Information). It can be seen that the spectrum obtained for the photoproduct in this time window corresponds to a photoproduct different from B (see Figure S3, Supporting Information), the final product of the irradiation identified by ${ }^{1} \mathrm{H}$ NMR spectroscopy. The photo product absorption in this time window resembles the features of the absorption spectrum of the Cc isomer (Figure S3, Supporting Information); unfortunately, it is not possible to obtain the spectrum of short lived $\mathrm{Cc}$ in acetonitrile, and the comparison must be made with the spectrum of $\mathbf{C c}$ in water. ${ }^{14}$ For the stated reasons, the photoproduct is attributed to the formation of $\mathbf{C c}$ through deactivation of $\mathbf{R}$ together with $\mathbf{C t}$. On this basis, the most reliable alternative is the attribution of the transient absorption $\mathbf{R}$ to a twisted excited state $\mathbf{P}^{*}$ corresponding to a rotation of $90^{\circ}$ around the isomerizing double bond, which, in 16 ns, yields both $\mathbf{C c}$ and $\mathbf{C t}$ with equal relaxation rates, as deduced from the bleaching intensities immediately and $100 \mathrm{~ns}$ after excitation (Figure 3b).

This interpretation is complemented by a kinetic analysis performed on the millisecond time scale at $330 \mathrm{~nm}$, showing that the resulting cis chalcone evolves to the chromene $\mathbf{B}$ through a fast tautomerization process $(\tau=10 \mathrm{~ms})$, in agreement with similar kinetics recorded previously ${ }^{17}$ for this type of system (Figure S4, Supporting Information).

The thermal stability of the final photoproduct, $\mathbf{B}$, in acetonitrile accounts for the absence of $\mathbf{C c}$ in the final solution; that is, the $\mathbf{C c}$ isomer initially formed from $\mathbf{P}^{*}$ is completely converted to $\mathbf{B}$. The $\mathbf{C c}$ species could not be obtained, either thermally or upon irradiation.

Pertinent photophysical events, with kinetic parameters, leading to the formation of $\mathbf{B}$ are summarized in Scheme 3. Following light excitation, the Franck-Condon excited state rapidly populates the trans chalcone singlet excited state ${ }^{1} \mathbf{C t}^{*}$ (LE), which evolves into the twisted state ${ }^{1} \mathbf{P}^{*}(\tau=2 \mathrm{ps})$. This

Scheme 3. Jablonski Diagram Showing the Species at Both the Excited and Ground States Intervening in the Photochemistry of Compound $1(\mathrm{Ct})$ in $\mathrm{CH}_{3} \mathrm{CN}$ Solution

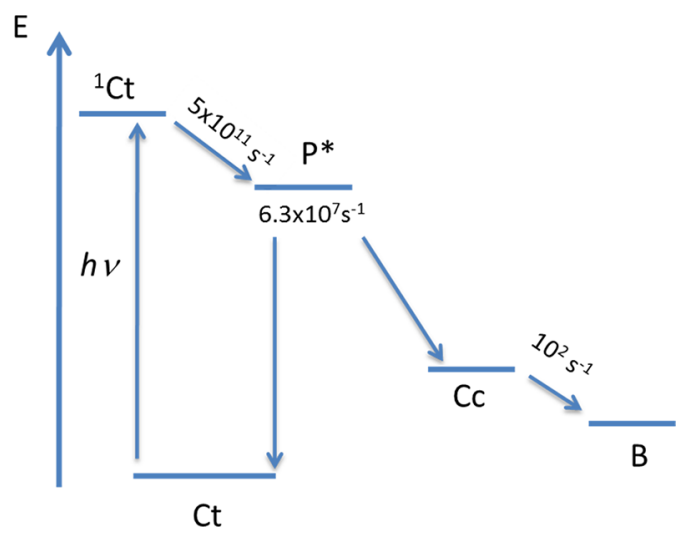



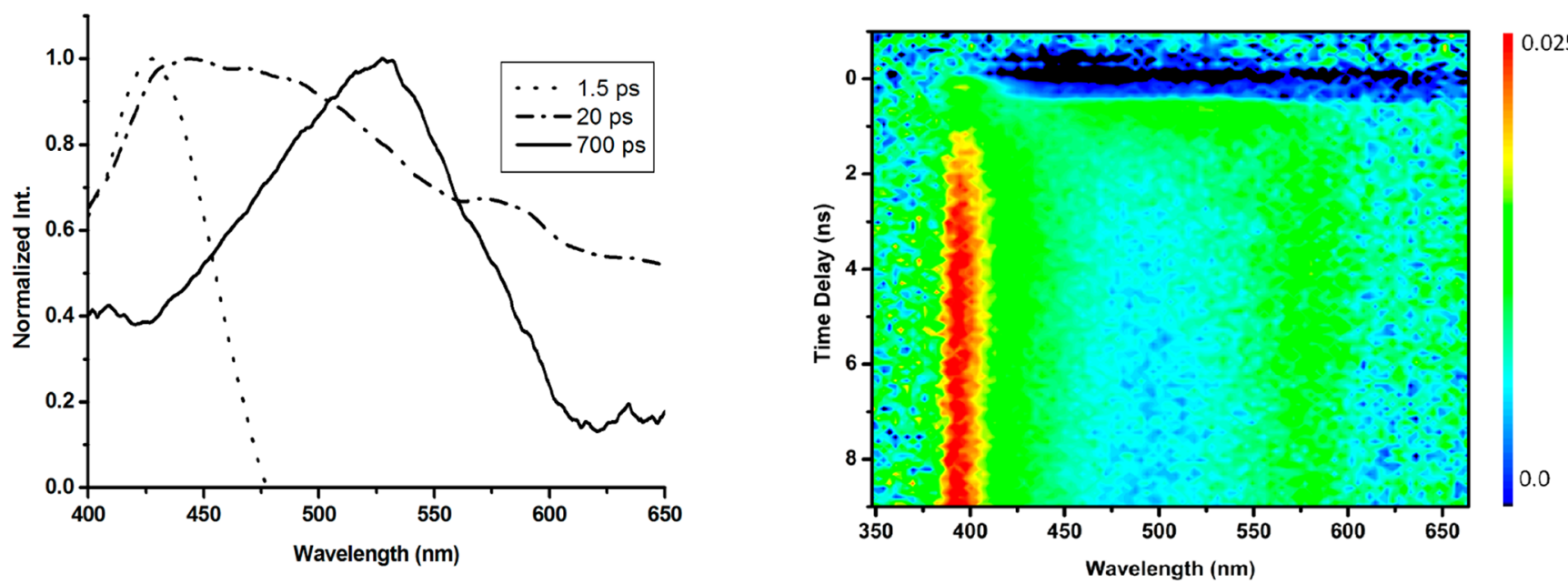

Figure 4. Ultrafast dynamics of 1 in $\mathrm{H}_{2} \mathrm{O} / \mathrm{CH}_{3} \mathrm{OH}$ at neutral $\mathrm{pH}$. Transient absorption spectra (left) on the picoseond time scale after 1.5, 40, and $700 \mathrm{ps}$ and (right) on the longer nanosecond time scale after $0-9 \mathrm{~ns}$. Colors correspond to $\Delta \mathrm{OD}$ values according to the included scale.

excited state is responsible for the formation of both $\mathbf{C t}$ and $\mathbf{C c}$ in the ground state.

The final product observed in the NMR experiment was formed through a tautomerization process in the ground state $(\tau=10 \mathrm{~ms}$ ) with a quantum yield of 0.1 (Figure 1 and Figure S4, Supporting Information).

Photochemistry in $\mathrm{H}_{2} \mathrm{O} / \mathrm{CH}_{3} \mathrm{OH}(1 / 1, v / v)$. The photo chemistry of 1 in the aqueous medium $\mathrm{H}_{2} \mathrm{O} / \mathrm{CH}_{3} \mathrm{OH}(1 / 1, \mathrm{v} /$ v) was previously studied. ${ }^{14}$ Irradiation of non equilibrated solutions $(100 \% \mathbf{C t})$ also leads to the exclusive formation of $\mathbf{B}$. The results from pump-probe experiments of $\mathbf{1}$ in $\mathrm{H}_{2} \mathrm{O}$ / $\mathrm{CH}_{3} \mathrm{OH}(1 / 1, \mathrm{v} / \mathrm{v})$ solution are presented in Figure 4 for two different temporal scales: picosecond and nanosecond.

An initial positive absorption at $420 \mathrm{~nm}$ appeared immediately after the excitation pulse and subsequently relaxed to a second red shifted broad band in the visible region with a maximum at $480 \mathrm{~nm}$. The initial spectral evolution is similar to that observed in previous experiments in $\mathrm{CH}_{3} \mathrm{CN}$ and can be assigned to the formation of the twisted ${ }^{1} \mathbf{P}^{*}$ state from ${ }^{1} \mathbf{C t}^{*}$ (LE state) with a lower kinetic rate constant ( $\tau=5 \mathrm{ps)} \mathrm{(Figure}$ S5, Supporting Information). The initial spectra of ${ }^{1} \mathbf{P}^{*}$ in the two media are identical. This signature is constant over time in acetonitrile solution, until the disappearance of the twisted excited state after 16 ns.

However, in aqueous solution, the maximum of the broad band further shifts from 480 to $520 \mathrm{~nm}$ (Figure 4, left). Unfortunately, the rate of disappearance of the $\mathbf{P}^{*}$ state could not be obtained because the signal was too weak in the ultrafast experiment, but on the other hand, no shift was observed in the longer time scale experiment, meaning that this process is probably faster than $50 \mathrm{ps}$. The aforementioned stabilization of the $S_{n} \leftarrow S_{1}$ transition of the twisted ${ }^{1} \mathbf{P}^{*}$ state from 480 to 520 $\mathrm{nm}$ (ca. $1600 \mathrm{~cm}^{-1}$ ), could correspond to water mediated tautomerization (enolization). We refer to the state absorbing at $520 \mathrm{~nm}$ as the $\mathbf{Q}^{*}$ state.

To confirm the involvement of proton transfer in the formation of the $\mathbf{Q}^{*}$ state, pump-probe experiments of $\mathbf{1}$ in $\mathrm{D}_{2} \mathrm{O} / \mathrm{CD}_{3} \mathrm{OD}(1 / 1, \mathrm{v} / \mathrm{v})$ solution were performed (Figure S6, Supporting Information). In $\mathrm{D}_{2} \mathrm{O} / \mathrm{CD}_{3} \mathrm{OD}$, the $\mathbf{P}^{*}$ state evolves into the $\mathrm{Q}^{*}$ state after ca. $100 \mathrm{ps}$, and even though the signal is still too weak in the ultrafast experiment, the spectral evolution can be clearly seen on the longer time scales (see Figure S6, Supporting Information). The decrease in the
$Q^{*}$ formation rate in the deuterated solvent mixture is a clear indication that proton migration is involved in the $\mathbf{P}^{*} \rightarrow \mathbf{Q}^{*}$ conversion, supporting the hypothesis of water mediated tautomerization. The $\mathbf{Q}^{*}$ state, which has a short lifetime ( 900 ps in $\mathrm{H}_{2} \mathrm{O} / \mathrm{CH}_{3} \mathrm{OH}, 3$ ns in $\mathrm{D}_{2} \mathrm{O} / \mathrm{CD}_{3} \mathrm{OD}$ ) evolves into a transient absorption extending over the whole visible region with two different maxima at 395 and $580 \mathrm{~nm}$ that does not decay in this time window (Figure 4, right). The kinetic traces collected at $580 \mathrm{~nm}$ showed a single exponential formation with a rate identical to the decay rate of the $Q^{*}$ state.

To clarify the subsequent process(es), flash photolysis experiments were performed between 280 and $700 \mathrm{~nm}$ (Figure $5)$.

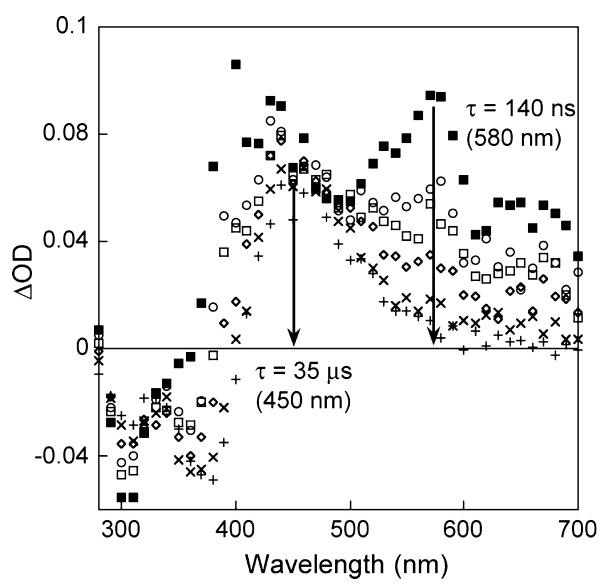

Figure 5. Transient absorption spectra of compound $\mathbf{1}$ in $\mathrm{H}_{2} \mathrm{O}$ / $\mathrm{CH}_{3} \mathrm{OH}(1 / 1, \mathrm{v} / \mathrm{v})$ at neutral $\mathrm{pH}$ on the nano to microsecond time scale from $15 \mathrm{~ns}$ to $0.9 \mu \mathrm{s}$. The evolution of the remaining signal is not

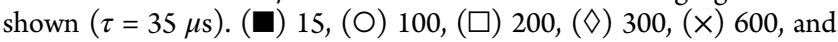
(+) $900 \mathrm{~ns}$.

The evolution on the nano /microsecond time scale allowed identification of two different contributions in addition to the negative signal clearly identified as the photobleaching of $\mathbf{C t}$. The previously observed transient at 580 and $395 \mathrm{~nm}$ decays according to a single exponential process with a rate equal to $7.3 \times 10^{6} \mathrm{~s}^{-1}(\tau=140 \mathrm{~ns})$, which could be affected by molecular oxygen as expected for a triplet state. After the triplet 
decayed, the remaining spectrum could be fitted by super imposing the spectra of $\mathbf{C t}$ and $\mathbf{C t}^{-}$(Figure S7, Supporting Information, and Figure 5). $\mathbf{C t}^{-}$(transient absorbing at 450 $\mathrm{nm}$ ) further decays as a single exponential with a rate of $2.9 \times$ $10^{4} \mathrm{~s}^{-1}(\tau=35 \mu \mathrm{s})$. The assignment to $\mathbf{C t}^{-}$is further corroborated by an observed dependence of the decay rate on the $\mathrm{pH}$ (and independence of the presence of oxygen). Whereas it is clear that part of the $\mathbf{C t}$ recovery is linked to the triplet decay, it is not straightforward, because of the spectral overlap, to elucidate whether $\mathbf{C t}^{-}$is formed initially from the deprotonation of $\mathbf{Q}^{*}$ or later from the deprotonation of the triplet state. However, the isotope effect observed in the rate of disappearance of $Q^{*}$ (900 ps in $\mathrm{H}_{2} \mathrm{O} / \mathrm{CH}_{3} \mathrm{OH}, 3 \mathrm{~ns}$ in $\mathrm{D}_{2} \mathrm{O}$ / $\mathrm{CD}_{3} \mathrm{OD}$ ) is compatible with a deprotonation reaction and, as a consequence, with the formation of $\mathbf{C t}^{-}$from $\mathbf{Q}^{*}$. Inspection of Figure 5 shows that the transient corresponding to $\mathbf{C t}^{-}$does not change during this time scale, which is also compatible with its formation from the deprotonation of the $\mathbf{Q}^{*}$ state. In fact, the subtraction of $\mathbf{C t}$ and $\mathbf{C t}^{-}$contributions at $2 \mu \mathrm{s}$ from the transient spectra obtained at successive times (Figure S8, Supporting Information) yields the disappearance of the same spectrum that we assigned to the pure triplet state.

Nevertheless, the final photoproduct $(\mathbf{C} \mathbf{c}$ or $\mathbf{B})$ is produced in a very small amount, either from $\mathbf{Q}^{*}$ or from the triplet. Also, the photochemical quantum yield in methanol/water $(0.02)$ is much lower than that in acetonitrile (0.1), and this probably implies that formation of $\mathrm{Ct}^{-}$and the triplet compete with the photoproduct formation pathway. From the flash photolysis data, after the disappearance of $\mathbf{C t}^{-}$, only the presence of $\mathbf{C t}$ can be observed. To identify the nature of the photoproducts, photoirradiations (at $365 \mathrm{~nm}$ with a continuous source) were carried out in equilibrated solutions of $\mathbf{1}$ in $\mathrm{H}_{2} \mathrm{O} / \mathrm{CH}_{3} \mathrm{OH}(1 / 1$, $\mathrm{v} / \mathrm{v}$ ) whose composition is a mixture of $\mathbf{C t}, \mathbf{C c}$, and $\mathbf{B}$ in a ratio 8:1:1, as followed by ${ }^{1} \mathrm{H}$ NMR spectroscopy (Figure 6).
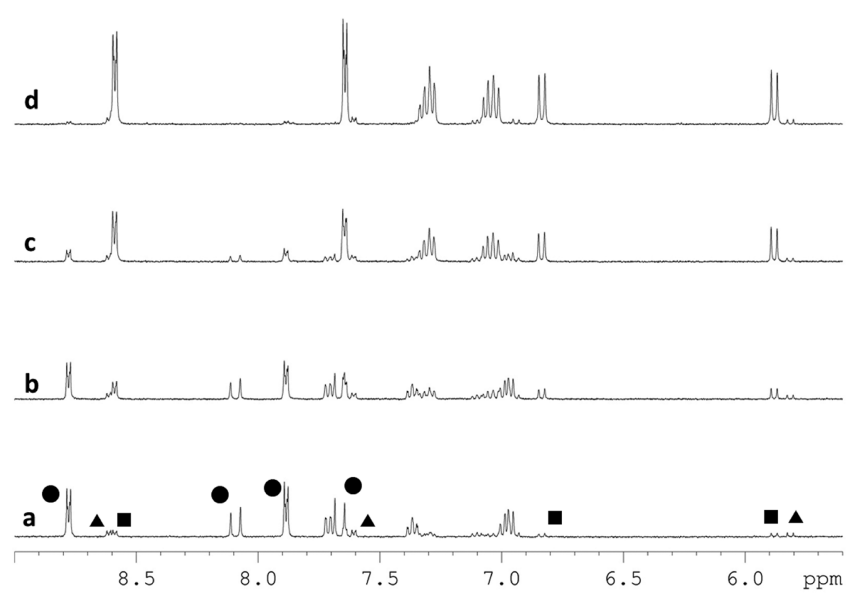

Figure 6. ${ }^{1} \mathrm{H}$ NMR spectra $(400 \mathrm{MHz}, 298 \mathrm{~K})$ of $\mathbf{1}$ in $\mathrm{D}_{2} \mathrm{O} / \mathrm{CD}_{3} \mathrm{OD}$ $(1: 1, \mathrm{v} / \mathrm{v})(\mathrm{a})$ at thermal equilibrium $[(\boldsymbol{\bullet}) 80 \% \mathbf{C t},(\boldsymbol{\Delta}) 10 \% \mathbf{C c},(\boldsymbol{\square})$ $10 \% \mathrm{~B}],(\mathrm{b}, \mathrm{c})$ during the course of the irradiation at $365 \mathrm{~nm}$, and $(\mathrm{d})$ after reaching the photostationary state upon irradiation at $365 \mathrm{~nm}$ ( $90 \%$ B, $10 \%$ Cc).

${ }^{1} \mathrm{H}$ NMR spectra were run before, during, and after reaching the photostationary state (Figure 6). The initial solution composed of $80 \% \mathbf{C t}, 10 \% \mathbf{C c}$, and $10 \% \mathrm{~B}$ is converted into a solution composed by $90 \%$ B and $10 \%$ Cc. The molar fraction of $\mathbf{C c}$ remains stable during the irradiation. The disappearance of $\mathbf{C t}$ and concomitant formation of $\mathbf{B}$ has a global quantum yield of 0.02 , which why it was not observed during flash photolysis.

If the photoproduct of the irradiation of $\mathbf{C t}$ were $\mathbf{C c}$, which, in a later step, would equilibrate with $\mathbf{B}$, then the ratio between $\mathbf{B}$ and $\mathbf{C c}$ would be constant. The ${ }^{1} \mathrm{H}$ NMR data clearly show that the photoproduct of the irradiation of $\mathbf{C t}$ is $\mathbf{B}$ and that the equilibrium between $\mathbf{B}$ and $\mathbf{C c}$ is not attained during the time scale of the experiment. ${ }^{14}$ In other words, $\mathbf{Q}^{*}$ yields $\mathbf{B}$ (in competition with triplet and $\mathbf{C t}^{-}$formation) whereas $\mathbf{P}^{*}$ yields Cc plus $\mathbf{C t}$. The formation of $\mathbf{B}$ in methanol/water solutions (and not $\mathbf{C c}$, as in acetonitrile) must be related with the nature of the new intermediary state $\mathbf{Q}^{*}$, which is present only in this solvent.

The formation of hydrogen bonds with water can facilitate the proton transfer between the two oxygen atoms, and the most straightforward assignment of $Q^{*}$ is the quinoidal tautomer derived from proton transfer (Scheme 4).

\section{Scheme 4. Representation of the Two Distinct Solvent Dependent Mechanisms for $1^{a}$}
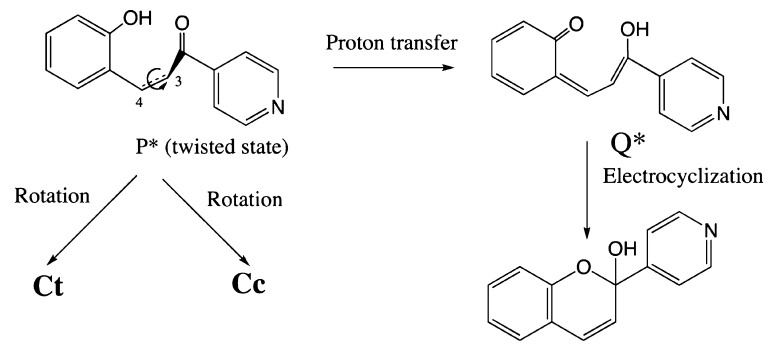

B

${ }^{a}$ In $\mathrm{CH}_{3} \mathrm{CN}$, the excited state $\mathbf{P}^{*}$ directly induces the formation of $\mathbf{C t}$ or $\mathbf{C c}$, whereas in $\mathrm{H}_{2} \mathrm{O} / \mathrm{CH}_{3} \mathrm{OH}, \mathbf{B}$ is promoted from the $\mathbf{Q}^{*}$ state formed after a solvent assisted proton transfer.

Whereas $\mathbf{P}^{*}$ can yield $\mathbf{C t}$ or $\mathbf{C c}$ through rotation around the $\mathrm{C} 3-\mathrm{C} 4$ bond, after the proton transfer, the most efficient reaction pathway for the quinone is electrocyclization to form B. The assignment of $\mathbf{Q}^{*}$ as a quinone is also in agreement with very efficient formation of the triplet, which was not previously observed in acetonitrile, where $\mathbf{P}^{*}$ was the only state observed.

A summary of the photophysical events with kinetic parameters leading to the formation of $\mathbf{B}$ in methanol/water is shown in Scheme 5. Following light excitation, the first steps are identical in acetonitrile and in water: The Franck-Condon excited state rapidly populates the trans chalcone singlet excited state ${ }^{1} \mathbf{C t}(\mathrm{LE})$, which evolves into the twisted state $\mathbf{P}^{*}(\tau=5$ ps). In $\mathrm{D}_{2} \mathrm{O} / \mathrm{CD}_{3} \mathrm{OD}$, this value reaches 5.4 ps. The state $\mathbf{P}^{*}$ evolves into $\mathbf{C t}^{-}$and into the transient $\mathbf{Q}^{*}$, which leads to $\mathbf{B}$ and triplets.

\section{CONCLUSIONS}

The photochromism of a 2 hydroxychalcone has been studied in $\mathrm{CH}_{3} \mathrm{CN}$ and in $\mathrm{H}_{2} \mathrm{O} / \mathrm{CH}_{3} \mathrm{OH}(1 / 1, \mathrm{v} / \mathrm{v})$ by steady state (UV-vis absorption, ${ }^{1} \mathrm{H}$ and ${ }^{13} \mathrm{C}$ NMR) and time resolved (ultrafast transient absorption and nanosecond flow flash photolysis) spectroscopies.

In the two types of media, the first step after excitation is the same and consists of the relaxation of the LE state to a twisted ${ }^{1} \mathbf{P} *$ state in a few picoseconds. In acetonitrile, this state deactivates to form $\mathbf{C c}$ and $\mathbf{C t}$ in identical proportions, the classic situation of double bond photoisomerization. The final 
Scheme 5. Jablonski Diagram Showing the Species at Both the Excited and Ground States Intervening in the Photochemistry of Compound $1(\mathrm{Ct})$ in $\mathrm{H}_{2} \mathrm{O} / \mathrm{CH}_{3} \mathrm{OH}(1 / 1$, v/v) Solution

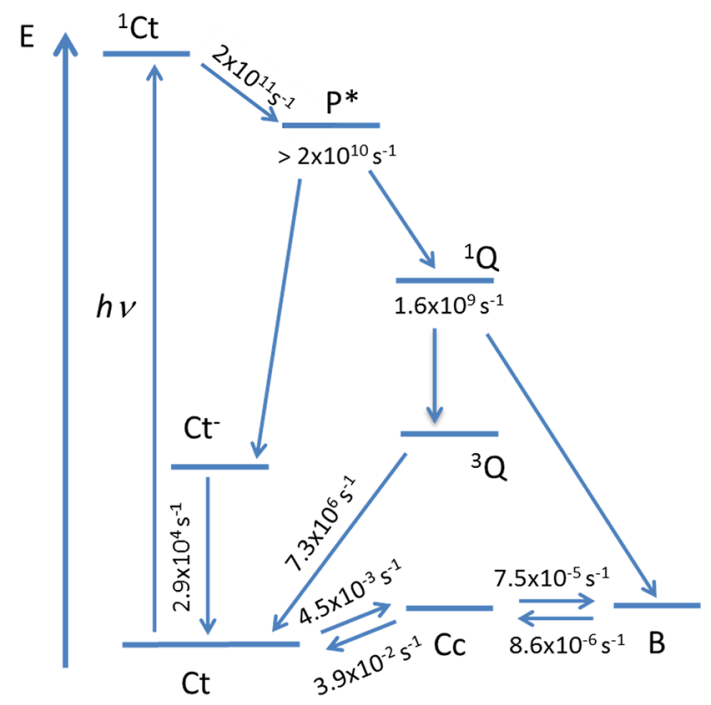

product, B, results from tautomerizaton of $\mathbf{C} \mathbf{c}$ with an observed quantum yield of 0.1

The presence of water, on the other hand, dramatically affects the photochemical pathway by promoting the formation of a tautomeric intermediate, between the ${ }^{1} \mathbf{P}^{*}$ state and the photoproducts. The main photochemical pathway from this tautomer is electrocyclization to form chromene $\mathbf{B}$, whereas all of the other pathways lead to $\mathbf{C t}$ (through $\mathbf{C t}^{-}$and ${ }^{3} \mathbf{Q}^{*}$ ); that is, no cis isomer is formed photochemically. However, the weight of the pathways leading back to $\mathbf{C t}$ is much larger than that of the pathways yielding $\mathbf{B}(\phi=0.02)$.

\section{AUTHOR INFORMATION}

\section{Corresponding Author}

*E mail: yoann.leydet@gmail.com (Y.L.), lima@fct.unl.pt (J.C.L.).

\section{Notes}

The authors declare no competing financial interest.

\section{ACKNOWLEDGMENTS}

This work was supported by the Fundação para a Ciência e Tecnologia, National NMR Network, through Projects PTDC/ QUI QUI/117996/2010 and SFRH/BPD/44230/2008 (Y.L.); the University of Bordeaux, Région Aquitaine; and the PHC Pessoa Franco Portuguese Programme.

(1) Dimmock, J. R.; Elias, D. W.; Beazely, M. A.; Kandepu, N. M. Curr. Med. Chem. 1999, 6, 1125-1149.

(2) Ono, M.; Watanabe, R.; Kawashima, H.; Yan, C.; Kimura, H.; Watanabe, H.; Haratake, M.; Saji, H.; Nakayama, M. J. Med. Chem. AEPEREACAS 6401.

(3) Bouas Laurent, H.; Dürr, H. Pure Appl. Chem. 2001, 73, 639665 .
(4) (a) Swain, T. In The Flavonoids; Harborne, J. B., Mabry, T. J., Mabry, H., Eds.; Chapman and Hall/CRC Press: London, 1975; pp 1096-1129. (b) Anthocyanins As Food Colors; Markakis, P., Ed.; Academic Press: New York, 1982. (c) Brouillard, R. O.; Dangles, O. In The Flavonoids: Advances in Research Since 1986; Harborne, J. B., Ed.; Chapman and Hall/CRC Press: New York, 1994; pp 565-588.

(5) (a) Yoshida, K.; Mori, M.; Kondo, T. Nat. Prod. Rep. 2009, 26, 884-915. (b) Goto, T.; Kondo, T. Angew. Chem., Int. Ed. Engl. 1991, 30, 17-33. (c) Yoshida, K.; Kondo, T.; Okazaki, Y.; Katou, K. Nature 1995, 373, 291. (d) Kondo, T.; Yoshida, K.; Nakagawa, K. A.; Kawai, T.; Tamura, H.; Goto, T. Nature 1992, 358, 515-518. (e) Brouillard, R.; Delaporte, B. J. Am. Chem. Soc. 1977, 99, 8461-8468. (f) Brouillard, R.; Delaporte, B.; Dubois, J. E J. Am. Chem. Soc. 1978, 100, 6202-6205. (g) Brouillard, R.; Lang, J. Can. J. Chem. 1990, 68, 755-761. (h) McClelland, R. A.; Gedge, S. J. Am. Chem. Soc. 1980, 102, 5838-5848. (i) McClelland, R. A.; McGall, G. H. J. Org. Chem. 1982, 47, 3730-3736.

(6) Pina, F.; Melo, M. J.; Laia, C. A. T.; Parola, A. J.; Lima, J. C Chem. Soc. Rev. 2012, 41, 869-908.

(7) Pina, F.; Melo, M. J.; Maestri, M.; Ballardini, R.; Balzani, V. J. Am. Chem. Soc. 1997, 119, 5556-5561.

(8) Pina, F.; Melo, M. J.; Maestri, M.; Passaniti, P.; Balzani, V. J. Am. Chem. Soc. 2000, 122, 4496-4498.

(9) Fedorova, O. A.; Gulakova, E. N.; Fedorov, Y. V.; Lobazova, I. E.; Alfimov, M. V.; Jonusauskas, G. J. Photochem. Photobiol. A 2008, 196, $239-243$.

(10) (a) Jiang, Y. B.; Wang, X. J.; Lin, L. J. Phys. Chem. 1994, 98, 12367-12372. (b) Rurack, K.; Dekhtyar, M. L.; Bricks, J. L.; Resch Genger, U.; Rettig, W. J. Phys. Chem. A 1999, 103, 9626-9635. (c) Rurack, K.; Bricks, J. L.; Reck, G.; Radeglia, R.; Resch Genger, U. J. Phys. Chem. A 2000, 104, 3087-3109. (d) Fayed, T. A.; Awad, M. K. Chem. Phys. 2004, 303, 317-326.

(11) Waldeck, D. H. Chem. Rev. 1991, 91, 415-436.

(12) Grabowski, Z. R.; Rotkiewicz, K.; Rettig, W. Chem. Rev. 2003, 103, 3899-4032.

(13) (a) Diniz, M.; Gomes, R.; Parola, A. J.; Laia, C. A. T.; Pina, F. J. Phys. Chem. B 2009, 113, 719-727. (b) Jiang, Y. B.; Wang, X. J.; Lin, L. J. Phys. Chem. 1994, 98, 12367-12372. (c) Rurack, K.; Dekhtyar, M. L.; Bricks, J. L.; Resch Genger, U.; Rettig, W. J. Phys. Chem. A 1999, 103, 9626-9635. (d) Rurack, K.; Bricks, J. L.; Reck, G.; Radeglia, R.; Resch Genger, U. J. Phys. Chem. A 2000, 104, 3087-3109. (e) Fayed, T. A.; Awad, M. K. Chem. Phys. 2004, 303, 317-326.

(14) Leydet, Y.; Parola, A. J.; Pina, F. Chem.-Eur. J. 2010, 16, 545555 .

(15) Leydet, Y.; Gavara, R.; Cunha Silva, L.; Parola, A. J.; Pina, F. Chem.-Eur. J. 2011, 17, 3663-3671.

(16) Hatchard, C. G.; Parker, C. A Proc. R. Soc. London, Ser. A 1956, 235, 518-536.

(17) Pina, F.; Maestri, M.; Balzani, V. In Handbook of Photochemistry and Photobiology; Nalwa, H. S., Ed.; American Science Publishers: Los Angeles, CA, 2003; Vol. 3, pp 411- 449. 